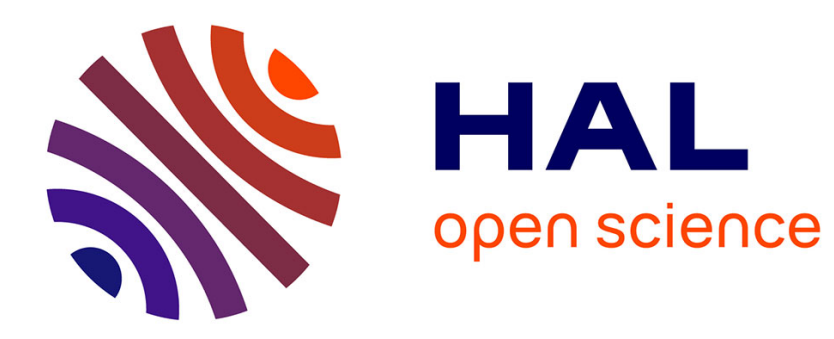

\title{
Boundary local null-controllability of the Kuramoto-Sivashinsky equation
}

Takéo Takahashi

\section{To cite this version:}

Takéo Takahashi. Boundary local null-controllability of the Kuramoto-Sivashinsky equation. Mathematics of Control, Signals, and Systems, 2017, 29 (2), 10.1007/s00498-016-0182-5 . hal-01373201

\section{HAL Id: hal-01373201 \\ https://hal.science/hal-01373201}

Submitted on 28 Sep 2016

HAL is a multi-disciplinary open access archive for the deposit and dissemination of scientific research documents, whether they are published or not. The documents may come from teaching and research institutions in France or abroad, or from public or private research centers.
L'archive ouverte pluridisciplinaire HAL, est destinée au dépôt et à la diffusion de documents scientifiques de niveau recherche, publiés ou non, émanant des établissements d'enseignement et de recherche français ou étrangers, des laboratoires publics ou privés. 


\title{
Boundary Local Null Controllability of the Kuramoto-Sivashinsky equation
}

\author{
Takéo Takahashi
}

April 25, 2016

\begin{abstract}
We prove that the Kuramoto-Sivashinsky equation is locally controllable in 1D and in 2D with one boundary control. Our method consists in combining several general results in order to reduce the nullcontrollability of this nonlinear parabolic equation to the exact controllability of a linear beam or plate system. This improves known results on the controllability of Kuramoto-Sivashinsky equation and gives a general strategy to handle the null-controllability of nonlinear parabolic systems.
\end{abstract}

Key words: Kuramoto-Sivashinsky equation, null controllability

2010 Mathematics Subject Classification: 93B05, 35K55, 93C10

\section{Introduction}

In this article, we study the boundary controllability of the Kuramoto-Sivashinsky equation, in space dimension 1 and 2. This equation is due to Kuramoto [12. (phase disturbance in chemical reaction system) and Sivashinsky [19] (model for a laminar flame). Lots of works are devoted to this equation: let us quote for instance [15, 16], [9], 11, etc. for the long time behavior. From a control point of view, this equation have been tackled in several papers: [10], 2], 4, [5, 7], etc. In particular, in 2], the author obtains the boundary null controllability of a linear system associated with the Kuramoto-Sivashinsky equation in 1D. In [4, the authors prove the local exact controllability to the trajectories of the Kuramoto-Sivashinsky equation in 1D by using boundary controls. In that case, they need two controls at the boundary and their method is based upon a Carleman estimate. Here our aim is to use only one control, in the 1D or in the 2D case. More precisely, we consider the control problem

$$
\left\{\begin{array}{l}
y_{t}+y_{x x x x}+a y_{x x}+y y_{x}=0, \quad x \in(0,1), t \in(0, T) \\
y(t, 0)=0, \quad y(t, 1)=0, \quad t \in(0, T), \\
y_{x}(t, 0)=u(t), \quad y_{x}(t, 1)=0, \quad t \in(0, T), \\
y(0, x)=y_{0}(x), \quad x \in(0,1) .
\end{array}\right.
$$

In the above system, the state $y$ is controlled by the function $u=u(t)$. One important step to study the controllability of 1.1 consists in studying the controllability of the linear system associated with (1.1). This was done in [2] and the author shows the null-controllability provided that $a \notin \mathcal{N}$, where

$$
\mathcal{N}:=\left\{\pi^{2}\left(k^{2}+\ell^{2}\right) ; k, \ell \in \mathbb{N}^{*}, k \neq \ell, k \equiv \ell \bmod 2\right\} .
$$

Our first main results shows the local null-controllability of 1.1 with the same hypothesis on $a$ :

Theorem 1.1. Assume that $a \notin \mathcal{N}$. Then the system 1.1 is locally null-controllable for any $T>0:$ there exists $c_{0}>0$ such that for any $\left\|y_{0}\right\|_{H^{-1}(0,1)} \leqslant c_{0}$, there exists $u \in L^{2}(0, T ; \mathbb{R})$ and a solution $y \in C\left([0, T] ; H^{-1}(0,1)\right)$ of (1.1) with

$$
y(T)=0 .
$$


The method of [2] is based on a precise spectral analysis, but does not allow to consider the nonlinear case. Nevertheless, we will use this spectral analysis in our proof, but in order to obtain a first result on an hyperbolic problem associated with (1.1).

Remark 1.2. The space for the initial conditions is different from [2] but in this paper, the author uses a control $u \in H^{1}(0, T)$.

Remark 1.3. If $a \in \mathcal{N}$, then the linear system is not exactly controllable and thus the method developed here can not be applied. Nevertheless, the nonlinear equation (1.1) can be null-controllable even if a $\in \mathcal{N}$. One can look at [1] and [3] for a similar problem in the case of the Korteweg-de Vries equation.

Our approach for the Kuramoto-Sivashinsky equation in 1D can be extended to the Kuramoto-Sivashinsky equation in $2 \mathrm{D}$, but the boundary conditions are different from the $1 \mathrm{D}$ case:

$$
\left\{\begin{array}{l}
y_{t}+\Delta^{4} y+a \Delta y+\frac{1}{2}|\nabla y|^{2}=0, \quad x \in \Omega, t \in(0, T) \\
y(t)=0, \quad x \in \partial \Omega, t \in(0, T) \\
\Delta y(t)=u 1_{\Gamma}, \quad x \in \partial \Omega, \quad t \in(0, T) \\
y(0, x)=y_{0}(x), \quad x \in \Omega
\end{array}\right.
$$

Our condition on $a$ is more restrictive in that case: let us consider the eigenvalues $\left(\lambda_{k}\right)_{k \geqslant 1}$ of the operator

$$
A_{0}:\left\{\phi \in H^{4}(\Omega) ; \phi=\Delta \phi=0 \quad \text { on } \partial \Omega\right\} \rightarrow L^{2}(\Omega), \quad \phi \mapsto \Delta^{4} \phi
$$

We consider that $\lambda_{1}$ is the smallest eigenvalue. We know that $\lambda_{1}>0$. Our second main result is

Theorem 1.4. Assume that $a<\sqrt{\lambda_{1}}$. Assume also one of the following assertions holds:

1. $\partial \Omega$ if of class $C^{5}$ and $\Gamma$ satisfies the geometric optics conditions;

2. $\Omega$ is a rectangle $(0, a) \times(0, b)$ and $\Gamma$ contains both a horizontal and a vertical segment of nonzero length.

Then the system 1.2 is locally null-controllable for any $T>0$ : there exists $c_{0}>0$ such that for any $\left\|y_{0}\right\|_{L^{2}(\Omega)} \leqslant$ $c_{0}$, there exists $u \in L^{2}\left(0, T ; L^{2}(\Gamma)\right)$ and a solution $y \in C\left([0, T] ; L^{2}(\Omega)\right)$ of $(1.2)$ with

$$
y(T)=0
$$

Remark 1.5. The conditions on $\Gamma$ and on a are sufficient conditions for the null-controllability of the linear system associated with (1.2). These conditions may not be necessary: we should expect for instance a condition on a as in Theorem 1.1 (not in a countable set) and for $\Gamma$ we should expect a condition as for the heat equation (non empty interior). However, to obtain such a result on $\Gamma$, one of the only methods is based on some Carleman estimates for a plate type system. Even in 1D, such a method fails to obtain only one control (see for instance [4]). As for Theorem 1.1, one could try to obtain the null-controllability of (1.2) even if the corresponding linear system is not controllable.

The proofs of Theorem 1.1 and Theorem 1.4 are based on several general results described in Section 3 . Using a result in [14, we can reduce the study to linear systems associated with (1.1) and (1.2). The corresponding linear systems are parabolic and to prove their null-controllability, we apply a method due to Russell [18, and consider the controllability or the observability of "hyperbolic" systems associated with them. Finally, in the 2D case, the observability of the corresponding plate system is obtained through a perturbation method developed in [6]. 


\section{Notation and preliminaries}

Let us consider a Hilbert space $\mathcal{X}$ and $A: \mathcal{D}(A) \rightarrow \mathcal{X}$ a generator of a strongly continuous semigroup $\left(e^{t A}\right)_{t \geqslant 0}$. Let us also consider a Hilbert space $U$ and $B \in \mathcal{L}\left(U, \mathcal{D}(A)^{*}\right)$ an operator. We consider the following control problem

$$
\dot{z}=A z+B u, \quad z(0)=z_{0} .
$$

We fix $\lambda_{\#} \in \rho(A)$. We can equip $\mathcal{D}(A)$ with the norm $\|z\|_{\mathcal{D}(A)}:=\left\|\left(\lambda_{\#}-A\right) z\right\|_{\mathcal{X}}$. Its dual $\mathcal{D}\left(A^{*}\right)^{*}$ with respect to pivot space $\mathcal{X}$ is the completion of $\mathcal{X}$ with respect to the norm $\|z\|_{\mathcal{D}\left(A^{*}\right)^{*}}:=\left\|\left(\lambda_{\#}-A\right)^{-1} z\right\|_{\mathcal{X}}$. We keep the notation $A$ and $\left(e^{t A}\right)_{t \geqslant 0}$. for the extensions of the generator to $\mathcal{X}$ and of the semigroup to $\mathcal{D}\left(A^{*}\right)^{*}$. Assume $T>0$. For $z_{0} \in \mathcal{D}\left(A^{*}\right)^{*}$ and $u \in L^{2}(0, T ; U)$, we can consider a solution $z$ of 2.1) as a mild solution

$$
z(t)=e^{t A} z_{0}+\int_{0}^{t} e^{(t-s) A} B u(s) d s .
$$

We say that $B$ is admissible if for any $z_{0} \in \mathcal{X}$ and for any $u \in L^{2}(0, T ; U)$, the above solution satisfies $z \in C([0, T] ; \mathcal{X})$. This is the case if for instance $A$ is self-adjoint and positive (parabolic case) and if $B \in$ $\mathcal{L}\left(U, \mathcal{D}\left(A^{1 / 2}\right)^{*}\right)$. In the case where $B$ is admissible, we say that the system (2.1) (or the pair $(A, B)$ ) is nullcontrollable in time $T$ if for any $z_{0} \in \mathcal{X}$, there exists $u \in L^{2}(0, T ; U)$ such that the solution of (2.1) verifies $z(T)=0$. We also say that the system 2.1 (or the pair $(A, B)$ ) is exactly controllable in time $T$ if for any $z_{0}, z_{1} \in \mathcal{X}$, there exists $u \in L^{2}(0, T ; U)$ such that $z(T)=z_{1}$. These two notions are related by duality to observability properties:

$$
\dot{\phi}=A^{*} \phi, \quad \psi=B^{*} \phi, \quad \phi(0)=\phi_{0} .
$$

For $\phi_{0} \in \mathcal{D}\left(A^{*}\right), \phi(t)=e^{t A^{*}} \phi_{0}$ and $\psi(t)=B^{*} e^{t A^{*}} \phi_{0}$. We say that the system (2.2) (or the pair $\left(A^{*}, B^{*}\right)$ ) is final-state observable in time $T$ if there exists $K(T)>0$ such that

$$
\left\|e^{T A^{*}} \phi_{0}\right\|_{\mathcal{X}}^{2} \leqslant K(T)^{2} \int_{0}^{T}\left\|B^{*} e^{t A^{*}} \phi_{0}\right\|_{U}^{2} d t \quad\left(\phi_{0} \in \mathcal{D}\left(A^{*}\right)\right) .
$$

We say that the system $(2.2)$ (or the pair $\left(A^{*}, B^{*}\right)$ ) is exactly observable in time $T$ if there exists $K(T)>0$ such that

$$
\left\|\phi_{0}\right\|_{\mathcal{X}}^{2} \leqslant K(T)^{2} \int_{0}^{T}\left\|B^{*} e^{t A^{*}} \phi_{0}\right\|_{U}^{2} d t \quad\left(\phi_{0} \in \mathcal{D}\left(A^{*}\right)\right) .
$$

The equivalence between observability and controllability is recalled in the proposition below, due to Dolecki and Russell [8] (see also Proposition 12.1.2 and Theorem 11.2.1 in [21]).

Proposition 2.1. Assume that $B$ is admissible. Then the pair $(A, B)$ is exactly controllable in time $T$ if and only if the pair $\left(A^{*}, B^{*}\right)$ is exactly observable in time $T$. The pair $(A, B)$ is null controllable in time $T$ if and only if the pair $\left(A^{*}, B^{*}\right)$ is final state observable in time T. Moreover, if the pair $\left(A^{*}, B^{*}\right)$ is exactly observable in time $T$, then there exists an operator $L_{T} \in \mathcal{L}(\mathcal{X}, U)$ such that a control for (2.1) is given by $u=L_{T} z_{0}$ and such that $\left\|L_{T}\right\|_{\mathcal{L}(\mathcal{X}, U)} \leqslant K(T)$.

\section{Some general results}

This section is devoted to the presentation of several important general results that are used in the proofs of Theorem 1.1 and Theorem 1.4

\subsection{The Russell method}

We recall here a method introduced by Russell [18] and that is completely described in 21] (Section 9.2). The idea is that the final state observability of a parabolic system can be obtained by the exact observability of 
an hyperbolic system associated with the parabolic system. More precisely, let us consider a Hilbert space $\mathcal{H}$ and a self-adjoint operator $A_{0}: \mathcal{D}\left(A_{0}\right) \rightarrow \mathcal{H}$, strictly positive. We also assume that $A_{0}^{-1}$ is compact. Let us also consider a Hilbert space $U$ and $C_{0} \in \mathcal{L}\left(\mathcal{D}\left(A_{0}^{1 / 2}\right), U\right)$ an operator. We consider the following observability problems

and

$$
\dot{z}=-A_{0} z, \quad y=C_{0} z, \quad z(0)=z_{0}
$$

$$
\ddot{w}=-A_{0} w, \quad y=C_{0} \dot{w}, \quad w(0)=w_{0}, \quad \dot{w}(0)=w_{1} .
$$

We denote by $\left(e^{-t A_{0}}\right)_{t \geqslant 0}$ the semigroup generated by $-A_{0}$. We introduce an orthonormal basis $\left(\varphi_{n}\right)_{n \in \mathbb{N}^{*}}$ in $\mathcal{H}$ consisting of eigenvectors of $A_{0}$ and $\left(\lambda_{n}\right)_{n \in \mathbb{N}^{*}}$ the corresponding eigenvalues. Then we have the following result:

Theorem 3.1. Assume that 3.2 is exactly observable in $\mathcal{D}\left(A_{0}^{1 / 2}\right) \times \mathcal{H}$ in time $T_{0}>0$ and that

$$
\sum_{n \in \mathbb{N}^{*}} \frac{1}{\lambda_{n}^{k}}<\infty
$$

for some $k \in \mathbb{N}^{*}$. Then (3.1) is final state observable in $\mathcal{H}$ for any time $T$ :

$$
\left\|e^{-A_{0} T} \varphi_{0}\right\|_{\mathcal{H}} \leqslant K(T)\left(\int_{0}^{T}\left\|C_{0} e^{-A_{0} t} \varphi_{0}\right\|_{U}^{2} d t\right)^{1 / 2},
$$

with

$$
K(T):=C e^{M / T} .
$$

This theorem is stated in this way in 21] (Theorem 9.2.2) but we give a sketch of the proof to precise the $\operatorname{cost} K(T)$ in 3.5 .

Proof. First, 3.2) is exactly controllable in $\mathcal{D}\left(A_{0}^{1 / 2}\right) \times \mathcal{H}$ in time $T_{0}>0$ means that the pair $(A, C)$ defined by

$$
A=\left[\begin{array}{cc}
0 & I \\
-A_{0} & 0
\end{array}\right], \quad C=\left[\begin{array}{ll}
0 & C_{0}
\end{array}\right]
$$

is exactly observable in $\mathcal{D}\left(A_{0}^{1 / 2}\right) \times \mathcal{H}$ in time $T_{0}>0$.

We define

$$
\mu_{k}=\operatorname{sgn}(k) \sqrt{\lambda_{|k|}} \quad\left(k \in \mathbb{Z}^{*}\right) .
$$

Then we use Lemma 9.2.1 in [21] and the exact observability of $(A, C)$ to obtain the existence of a bounded sequence $\left(F_{n}\right)_{n \in \mathbb{Z}^{*}}$ in $L^{2}\left(0, T_{0} ; U\right)$ that is biorthogonal in $L^{2}\left(0, T_{0} ; U\right)$ to the sequence $\left(e^{i \mu_{k} t} C_{0} \varphi_{k}\right)_{n \in \mathbb{Z}^{*}}$ in $L^{2}\left(0, T_{0} ; U\right)$.

The idea is to construct from $\left(F_{n}\right)_{n \in \mathbb{Z}^{*}}$ a sequence $\left(G_{n}\right)_{n \in \mathbb{N}^{*}}$ in $L^{2}(0, T ; U)$ biorthogonal to $\left(e^{-\lambda_{k} t} C_{0} \varphi_{k}\right)_{n \in \mathbb{N}^{*}}$. This is done in the proof of Theorem 9.2.2 in [21] (see formula (9.2.24), p.294). Using Lemma 9.2.3 in [21], we have

$$
\sum_{n \in \mathbb{N}^{*}} e^{-2 T \lambda_{n}}\left\|G_{n}\right\|_{L^{2}(0, T ; U)}^{2} \leqslant C \sum_{n \in \mathbb{N}^{*}} e^{-T \lambda_{n}}(\nu+1)^{2} e^{3 \nu / 2} e^{-\frac{\beta \lambda_{n}}{2 \sqrt{\nu+1}}}
$$

with $\delta>\max \left(0,2\left(T_{0}-\pi\right)\right), \beta \in\left(0, \frac{T}{2}\right)$ and $\nu=(\pi+\delta)^{2} / \beta$. The constant $C$ appearing in the above estimate only depends on $\delta$. Using that $e^{-x}<k ! / x^{k}$ for all $x>0$, we deduce that for all $k \in \mathbb{N}^{*}$, there exists $C=C(\delta, k)$ such that

$$
\sum_{n \in \mathbb{N}^{*}} e^{-2 T \lambda_{n}}\left\|G_{n}\right\|_{L^{2}(0, T ; U)}^{2} \leqslant C(\nu+1)^{2+3 k / 2} e^{3 \nu / 2} \sum_{n \in \mathbb{N}^{*}} \frac{1}{\lambda_{n}^{k}} .
$$

Gathering the above estimate, (3.3) and the above conditions on $\beta, \nu, \delta$, we deduce

$$
\sum_{n \in \mathbb{N}^{*}} e^{-2 T \lambda_{n}}\left\|G_{n}\right\|_{L^{2}(0, T ; U)}^{2} \leqslant C^{2} e^{2 M / T} .
$$

Applying Lemma 9.1.4 in [21, we deduce (3.4) and 3.5. 


\subsection{The source term method}

We recall a method introduced in [14] and that allows us to deal with the controllability of nonlinear parabolic systems. Assume $T>0$. In what follows, we consider the subset $\mathcal{R}_{T}$ of the functions $\rho$ satisfying the following properties

$$
\rho:[0, T] \rightarrow[0, \infty) \text { continuous, non increasing, positive on }[0, T), \rho(T)=0 .
$$

We denote by $L_{\rho}^{2}(0, T ; Y)$ the $L^{2}$ space with values in $Y$ and with measure $\lambda_{1} / \rho^{2}$ where $\lambda_{1}$ denotes the Lebesgue measure on $(0, T)$. We consider the same hypotheses as in the previous subsection: $\mathcal{H}$ is a Hilbert space, $A_{0}: \mathcal{D}\left(A_{0}\right) \rightarrow \mathcal{H}$ is a self-adjoint operator strictly positive such that $A_{0}^{-1}$ is compact. We also consider a Hilbert space $U$ and $B_{0} \in \mathcal{L}\left(U, \mathcal{D}\left(A_{0}^{1 / 2}\right)^{*}\right)$ an operator. To shorten the notation in this subsection, we write

$$
\mathcal{H}_{1 / 2}:=\mathcal{D}\left(A_{0}^{1 / 2}\right), \quad \mathcal{H}_{-1 / 2}:=\mathcal{D}\left(A_{0}^{1 / 2}\right)^{*} .
$$

In this subsection, we consider the following control problem

$$
\dot{z}=-A_{0} z+B_{0} u+f, \quad z(0)=z_{0}
$$

Theorem 3.2. Assume that for all $t>0$ we have the observability inequality

$$
\left\|e^{-A_{0} t} \varphi_{0}\right\|_{\mathcal{H}} \leqslant K(t)\left(\int_{0}^{t}\left\|B_{0}^{*} e^{-A_{0} t} \varphi_{0}\right\|_{U}^{2} d t\right)^{1 / 2},
$$

with $K:(0, \infty) \rightarrow(0, \infty)$ continuous and non increasing. There exist $\rho_{0}, \rho_{1} \in \mathcal{R}_{T}$ such that for any $z_{0} \in \mathcal{H}$ and $f \in L_{\rho_{1}}^{2}\left(0, T ; \mathcal{H}_{-1 / 2}\right)$, there exists $u \in L_{\rho_{0}}^{2}(0, T ; U)$ such that the solutions of (3.7) satisfies

$$
\|z\|_{L_{\rho_{0}}^{2}\left(0, T ; \mathcal{H}_{1 / 2}\right)}^{2}+\left\|\frac{z}{\rho_{0}}\right\|_{C^{0}([0, T] ; \mathcal{H})}^{2}+\|\dot{z}\|_{L_{\rho_{0}}^{2}\left(0, T ; \mathcal{H}_{-1 / 2}\right)}^{2}+\|u\|_{L_{\rho_{0}}^{2}(0, T ; U)}^{2} \leqslant C\left(\left\|z_{0}\right\|_{\mathcal{H}}+\|f\|_{L_{\rho_{1}}^{2}\left(0, T ; \mathcal{H}_{-1 / 2}\right)}^{2}\right) .
$$

In particular, (3.7) is null-controllable.

In the proof of this theorem, $\rho_{0}$ and $\rho_{1}$ are constructed in the following way: we consider $q>1$ and

$$
\rho_{0}(t):=\rho_{1}\left(q^{2}(t-T)+T\right) K((q-1)(T-t)) \quad\left(t \in\left[T\left(1-\frac{1}{q^{2}}\right), T\right]\right),
$$

and $\rho_{0}$ extended on $\left[0, T\left(1-\frac{1}{q^{2}}\right)\right]$ so that $\rho_{0} \in \mathcal{R}_{T}$.

The above statement is slightly different from the result in [14] since in our case $B_{0}^{*}$ is not a bounded operator of $\mathcal{H}$. The proof is nevertheless completely similar, we only remind the main steps of proof for sake of completeness.

Sketch of the proof of Theorem 3.2. We set

$$
T_{k}:=T-\frac{T}{q^{k}} \quad(k \in \mathbb{N}) .
$$

From 3.10, , we have the relation

$$
\rho_{0}\left(T_{k+2}\right)=\rho_{1}\left(T_{k}\right) K\left(T_{k+2}-T_{k+1}\right)
$$

Then we define the following sequence: $a_{0}:=z_{0}$ and for any $k \geqslant 0, a_{k+1}:=z_{1}\left(T_{k+1}^{-}\right)$where $z_{1}$ is the solution of the system

$$
\left\{\begin{array}{l}
\dot{z}_{1}=-A z_{1}+f \quad \text { in }\left(T_{k}, T_{k+1}\right), \\
z_{1}\left(T_{k}^{+}\right)=0 .
\end{array}\right.
$$


Using the classical results for parabolic system, we have for all $k \geqslant 0$,

$$
\left\|a_{k+1}\right\|_{\mathcal{H}}^{2} \leqslant C\|f\|_{L^{2}\left(T_{k}, T_{k+1} ; \mathcal{H}_{-1 / 2}\right)}^{2} .
$$

where the constant $C$ is independent of $k$.

Finally, we consider the control system

$$
\left\{\begin{array}{l}
\dot{z}_{2}=-A z_{2}+B u \quad \text { in }\left(T_{k}, T_{k+1}\right) \\
z_{2}\left(T_{k}^{+}\right)=a_{k}
\end{array}\right.
$$

From 23.8 and Proposition 2.1, there exists a control $u \in L^{2}\left(T_{k}, T_{k+1} ; U\right)$ such that

$$
z_{2}\left(T_{k+1}^{-}\right)=0
$$

and

$$
\|u\|_{L^{2}\left(T_{k}, T_{k+1} ; U\right)}^{2} \leqslant K^{2}\left(T_{k+1}-T_{k}\right)\left\|a_{k}\right\|_{\mathcal{H}}^{2} .
$$

We then define $z=z_{1}+z_{2}$ and using the relations of $z_{1}$ and $z_{2}$ at $T_{k}^{-}$and $T_{k}^{+}$, we can check that $z$ is solution of (3.7). Moreover, from (3.11), (3.13) and (3.15), we deduce that for all $k \geqslant 0$,

$$
\|u\|_{L_{\rho_{0}}^{2}\left(T_{k+1}, T_{k+2} ; U\right)}^{2} \leqslant C\|f\|_{L_{\rho_{1}}^{2}\left(T_{k}, T_{k+1} ; \mathcal{H}_{-1 / 2}\right)}^{2} .
$$

From the classical results for parabolic system, we have for any $k \geqslant 0$,

$$
\begin{aligned}
&\|z\|_{L^{2}\left(T_{k+1}, T_{k+2} ; \mathcal{H}_{1 / 2}\right)}^{2}+\|z\|_{C^{0}\left(\left[T_{k+1}, T_{k+2}\right] ; \mathcal{H}\right)}^{2}+\|\dot{z}\|_{L^{2}\left(T_{k+1}, T_{k+2} ; \mathcal{H}_{-1 / 2}\right)}^{2} \\
& \leqslant C\left(\left\|a_{k+1}\right\|_{\mathcal{H}}^{2}+\|u\|_{L^{2}\left(T_{k+1}, T_{k+2} ; U\right)}^{2}+\|f\|_{L^{2}\left(T_{k+1}, T_{k+2} ; \mathcal{H}_{-1 / 2}\right)}^{2}\right)
\end{aligned}
$$

Thus, from the above relation, from $(3.13)$ and from $(3.15)$, we deduce that for all $k \geqslant 0$,

$$
\|z\|_{L^{2}\left(T_{k+1}, T_{k+2} ; \mathcal{H}_{1 / 2}\right)}^{2}+\|z\|_{C^{0}\left(\left[T_{k+1}, T_{k+2}\right] ; \mathcal{H}\right)}^{2}+\|\dot{z}\|_{L^{2}\left(T_{k+1}, T_{k+2} ; \mathcal{H}_{-1 / 2}\right)}^{2} \leqslant C \rho_{0}\left(T_{k+2}\right)^{2}\|f\|_{L_{\rho_{1}}^{2}\left(T_{k}, T_{k+2} ; \mathcal{H}_{-1 / 2}\right)}^{2} .
$$

Since $\rho_{0}$ is non increasing we deduce

$$
\|z\|_{L_{\rho_{0}}^{2}\left(T_{k+1}, T_{k+2} ; \mathcal{H}_{1 / 2}\right)}^{2}+\left\|\frac{z}{\rho_{0}}\right\|_{C^{0}\left(\left[T_{k+1}, T_{k+2}\right] ; \mathcal{H}\right)}^{2}+\|\dot{z}\|_{L_{\rho_{0}}^{2}\left(T_{k+1}, T_{k+2} ; \mathcal{H}_{-1 / 2}\right)}^{2} \leqslant C\|f\|_{L_{\rho_{1}}^{2}\left(T_{k}, T_{k+2} ; \mathcal{H}_{-1 / 2}\right)}^{2} .
$$

A similar estimate is valid for $\left(T_{0}, T_{1}\right)$ and thus we deduce (3.9).

\subsection{The Perturbation method}

In this subsection, we recall some results obtained in [6] in order to deduce the exact observability of

$$
\ddot{w}=-A_{0} w+a A_{0}^{1 / 2} w, \quad y=C_{0} v, \quad w(0)=w_{0}, \quad \dot{w}(0)=w_{1}
$$

from the exact observability of

$$
\ddot{w}=-A_{0} w, \quad y=C_{0} v, \quad w(0)=w_{0}, \quad \dot{w}(0)=w_{1},
$$

where as the previous subsections, $\mathcal{H}$ is a Hilbert space, $A_{0}: \mathcal{D}\left(A_{0}\right) \rightarrow \mathcal{H}$ is a strictly positive self-adjoint operator such that $A_{0}^{-1}$ is compact. We have denoted by $A_{0}^{1 / 2}$ the square root of $A_{0}$ (see for instance [21, p.81]). We also consider a Hilbert space $U$ and $B_{0} \in \mathcal{L}\left(U, \mathcal{D}\left(A_{1}^{1 / 2}\right)^{*}\right)$ an admissible operator (see Section 2).

We denote by $\lambda_{n}, n \in \mathbb{N}^{*}$, the eigenvalues of $A_{0}$. They are real positive and we denote by $\lambda_{1}$ the smallest one.

We have the following result.

Theorem 3.3. Assume that $(3.21)$ is exactly observable and $a<\sqrt{\lambda_{1}}$. Then $\sqrt{3.20}$ is exactly observable.

This result is stated [6. Proposition 3.1]. The proof is done in the case where $C_{0}$ is a bounded operator (i.e. $\left.C_{0} \in \mathcal{L}(\mathcal{H}, U)\right)$ but this assumption is not used. Note that the time of exact observability is not obtained in this theorem, and this is due to the method of proof based on a spectral method (see [17]). 


\section{Controllability of the Kuramoto-Sivashinsky equation in 1D}

We are now using the result of the previous section in order to obtain the null-controllability of the KuramotoSivashinsky equation in $1 \mathrm{D}$.

\subsection{Abstract framework}

First we introduce operators in order to rewrite 1.1 .

We define

$$
\begin{gathered}
\mathcal{D}\left(A_{0}\right)=\left\{y \in H^{4}(0,1) ; y=y_{x}=0 \quad \text { in }\{0,1\}\right\}, \\
A_{0}: \mathcal{D}\left(A_{0}\right) \rightarrow L^{2}(0,1), \quad y \mapsto y_{x x x x}+a y_{x x} .
\end{gathered}
$$

The operator $A_{0}$ is self-adjoint and $-A_{0}$ is the infinitesimal generator of a parabolic semigroup $\left(e^{-t A_{0}}\right)_{t \geqslant 0}$.

In what follows, we consider $\lambda_{\#} \in \rho\left(A_{0}\right)$ such that $A_{\#}:=\lambda_{\#}+A_{0}$ is positive and we set

$$
\mathcal{H}^{s}:=\mathcal{D}\left(A_{\#}^{s / 4}\right) \quad(s \geqslant 0), \quad \mathcal{H}^{-s}:=\mathcal{D}\left(A_{\#}^{s / 4}\right)^{\prime} \quad(s \geqslant 0),
$$

where $\mathcal{X}^{\prime}$ denotes the dual of $\mathcal{X}$ with respect to the space $L^{2}(0,1)$.

We can consider $A_{0}$ as an operator

$$
A_{0}: \mathcal{H}^{s+4} \rightarrow \mathcal{H}^{s}, \quad(s \in \mathbb{R})
$$

In particular,

$$
A_{\#}: \mathcal{H}^{s+4} \rightarrow \mathcal{H}^{s}, \quad(s \in \mathbb{R})
$$

is a unitary transformation.

In order to obtain a weak formulation for system (1.1), we multiply formally the first equation of (1.1) by $\varphi \in \mathcal{H}^{4}$. After some integrations by parts, we obtain

$$
\frac{d}{d t} \int_{0}^{1} y \varphi d x+\int_{0}^{1} y A_{0} \varphi d x-\int_{0}^{1}\left(\frac{y^{2}}{2}\right) \varphi_{x} d x=u(t) \varphi_{x x}(t, 0) .
$$

In order to write the corresponding control operator, we use a classical approach and define the operator $D_{0}: U:=\mathbb{R} \rightarrow \mathcal{H}^{1}=H_{0}^{1}(0,1)$ as follows: $D_{0} u=y$ is the solution of

$$
\left\{\begin{array}{l}
\lambda_{\#} y+\left(y_{x x x x}+a y_{x x}\right)=0, \quad x \in(0,1) \\
y(0)=y(1)=0 \\
y_{x}(0)=u, \quad y_{x}(1)=0 .
\end{array}\right.
$$

More precisely, $D_{0} u$ is the unique element of $\mathcal{H}^{1}=H_{0}^{1}(0,1)$ such that

$$
\left\langle D_{0} u, f\right\rangle_{\mathcal{H}^{1}, \mathcal{H}^{-1}}=u\left(A_{\#}^{-1} f\right)_{x x}(0) \text {. }
$$

Since $A_{\#}^{-1}\left(\mathcal{H}^{-1}\right)=\mathcal{H}^{3} \subset H^{3}(0,1), D_{0} u$ is well-defined and $D_{0} \in \mathcal{L}\left(U, \mathcal{H}^{1}\right)$.

Then, we define the operator $B_{0}$ by

$$
B_{0}:=A_{\#} D_{0}: U=\mathbb{R} \rightarrow \mathcal{H}^{-3} .
$$

One can check that for all $\varphi \in \mathcal{H}^{3}$ and $u \in \mathbb{R}$,

$$
\left\langle B_{0} u, \varphi\right\rangle_{\mathcal{H}^{-3}, \mathcal{H}^{3}}=u \varphi_{x x}(0) .
$$

Finally, we define $F: L^{2}(0,1) \rightarrow \mathcal{H}^{-2}$ by

$$
\langle F(y), \varphi\rangle_{\mathcal{H}^{-2}, \mathcal{H}^{2}}:=-\int_{0}^{1}\left(\frac{y^{2}}{2}\right) \varphi_{x} d x \quad\left(\varphi \in \mathcal{H}^{2}\right) .
$$


The function $F$ is well-defined since $H^{1}(0,1) \subset L^{\infty}(0,1)$.

The above definitions allow us to write (4.4) as

$$
y_{t}+A_{0} y+F(y)=B_{0} u,
$$

and if $u \in L^{2}(0, T)$, we consider solutions satisfying the following regularity

$$
y \in H^{1}\left(0, T ; \mathcal{H}^{-3}\right) \cap L^{2}\left(0, T ; \mathcal{H}^{1}\right) \cap C\left([0, T] ; \mathcal{H}^{-1}\right) .
$$

Note that if $y$ satisfies 4.10 , then

$$
y \in L^{4}\left(0, T ; L^{2}(0,1)\right)
$$

and in particular $F(y) \in L^{2}\left(0, T ; \mathcal{H}^{-2}\right)$.

\subsection{Controllability of the linear problem}

Using the previous section, we can write

$$
\left\{\begin{array}{l}
y_{t}+y_{x x x x}+a y_{x x}=0, \quad x \in(0,1), \quad t \in(0, T) \\
y(t, 0)=0, \quad y(t, 1)=0, \quad t \in(0, T), \\
y_{x}(t, 0)=u(t), \quad y_{x}(t, 1)=0, \quad t \in(0, T), \\
y(0, x)=y_{0}(x), \quad x \in(0,1) .
\end{array}\right.
$$

as

$$
y_{t}+A_{0} y=B_{0} u, \quad y(0)=y_{0} .
$$

The main result of this section is the following result.

Theorem 4.1. Assume $a \notin \mathcal{N}$. For $y_{0} \in \mathcal{H}^{-1}$, there exists $u \in L^{2}(0, T)$ such that $y(T)=0$. Moreover, there exists an operator $L_{T} \in \mathcal{L}\left(\mathcal{H}^{-1}, \mathbb{R}\right)$ such that $u$ can be given by $u=L_{T}\left(y_{0}\right)$. We have

$$
\left\|L_{T}\right\|_{\mathcal{L}\left(\mathcal{H}^{-1}, \mathbb{R}\right)} \leqslant K(T)
$$

with

$$
K(T):=C e^{M / T},
$$

for some positive constants $C, M$.

Proof of Theorem 4.1. First, we show that the adjoint of $B_{0}$ defined by 4.7) is

$$
C_{0}:=B_{0}^{*}: \mathcal{H}^{1} \rightarrow \mathbb{R}, \varphi \mapsto\left(A_{\#}^{-1 / 2} \varphi\right)_{x x}(0) .
$$

Indeed, considering $A_{0}$ as an operator of $\mathcal{H}^{-1}$ with domain $D\left(A_{0}\right)=\mathcal{H}^{3}$, we have $D\left(A_{0}^{1 / 2}\right)=\mathcal{H}^{1}$ and the dual $D\left(A_{0}^{1 / 2}\right)^{*}$ of $D\left(A_{0}^{1 / 2}\right)$ with respect to $\mathcal{H}^{-1}$ is $D\left(A_{0}^{1 / 2}\right)^{*}=\mathcal{H}^{-3}$ and

$$
\left\langle B_{0} u, \varphi\right\rangle_{D\left(A_{0}^{1 / 2}\right)^{*}, D\left(A_{0}^{1 / 2}\right)}=\left\langle A_{\#}^{1 / 2} D_{0} u, \varphi\right\rangle_{\mathcal{H}^{-1}, \mathcal{H}^{1}}=u\left(A_{\#}^{-1 / 2} \varphi\right)_{x x}(0) .
$$

where we have used $(4.6)$.

Second, we show that $\left(-A_{0}, C_{0}\right)$ is final state observable in any time $T>0$ with

$$
A_{0}: \mathcal{H}^{4} \rightarrow L^{2}(0,1) \quad \text { and } \quad C_{0}: \mathcal{H}^{2} \rightarrow \mathbb{R}
$$

the last operator being the restriction of $C_{0}$ defined by 4.13 to $\mathcal{H}^{2}$. In order to do that, we are going to use Theorem 3.1 and thus to consider the exact observability in $\mathcal{H}^{2} \times \mathcal{H}$ of

$$
\left\{\begin{array}{l}
\ddot{w}=-A_{0} w \\
y=C_{0} \dot{w} .
\end{array}\right.
$$


Using Lemma 2.1 in [2], we have that if $a \notin \mathcal{N}$, then the eigenvalues $\left(\lambda_{k}\right)$ of $A_{0}$ are simple and using Lemma 2.2 in 2 , we also have that

$$
\lambda_{k} \sim D_{1} k^{4}, \quad D_{1}>0 .
$$

The two properties imply that the eigenvalues admit a gap: there exists $\gamma>0$ such that

$$
\lambda_{k+1}-\lambda_{k} \geqslant \gamma \quad(k \in \mathbb{N}) .
$$

Let us denote by $\left(\phi_{k}\right)$ a family of eigenvectors of $A_{0}$ associated to $\left(\lambda_{k}\right)$ and forming an orthonormal basis of $\mathcal{H}$. We show the exact observability of (4.14) by applying a spectral method: using Proposition 4.5 in [17, we have that (4.14) is exactly observable in some time $T_{0}$ if and only if there exists $\delta>0$ such that

$$
\left|C_{0} \phi_{k}\right| \geqslant \delta \quad(k \in \mathbb{N}) .
$$

From 4.13,

$$
C_{0} \phi_{k}=\frac{\left(\phi_{k}\right)^{\prime \prime}(0)}{\sqrt{\lambda_{\#}+\lambda_{k}}}
$$

and using Lemma 2.1 and Lemma 2.2 in [2], we deduce that $C_{0} \phi_{k} \neq 0$ and

$$
\lim _{k \rightarrow \infty}\left|C_{0} \phi_{k}\right|=d_{1}>0 .
$$

This implies (4.16) and thus the exact observability of (4.14) for some time $T_{0}>0$.

Now, from (4.15), we deduce 3.3 and thus applying Theorem 3.1 we obtain

$$
\left\|e^{-A_{0} T} \varphi_{0}\right\|_{\mathcal{H}} \leqslant K(T)\left(\int_{0}^{T}\left\|B_{0}^{*} e^{-A_{0} t} \varphi_{0}\right\|_{U}^{2} d t\right)^{1 / 2} \quad\left(\varphi_{0} \in \mathcal{H}^{1}\right)
$$

and there exist $C$ and $M$ such that

$$
K(T):=C e^{M / T} .
$$

This implies in particular

$$
\left\|e^{-A_{0} T} \varphi_{0}\right\|_{\mathcal{H}^{-1}} \leqslant K(T)\left(\int_{0}^{T}\left\|B_{0}^{*} e^{-A_{0} t} \varphi_{0}\right\|_{U}^{2} d t\right)^{1 / 2} \quad\left(\varphi_{0} \in \mathcal{H}^{1}\right) .
$$

From Proposition 2.1, we deduce that (4.11) is null-controllable for any time $T>0$ in $\mathcal{H}^{-1}$ : for any $y_{0} \in \mathcal{H}^{-1}$, there exists $u=L_{T}\left(y_{0}\right) \in L^{2}(0, T ; U)$ with

$$
\|u\|_{L^{2}(0, T ; \mathbb{R})} \leqslant K(T)\left\|y_{0}\right\|_{\mathcal{H}^{-1}}
$$

such that $y(T)=0$.

\subsection{Proof of Theorem 1.1}

We are now in position to prove Theorem 1.1

We recall that $K$ satisfies 4.12 . We take

$$
\begin{gathered}
q \in\left(1,2^{1 / 4}\right), \quad \alpha>\frac{M T q^{4}}{2(q-1)}, \\
\rho_{1}(t)=\exp \left(-\frac{\alpha}{(T-t)^{2}}\right) .
\end{gathered}
$$


We can define $\rho_{0}$ as

$$
\rho_{0}(t)=C \exp \left(-\frac{\alpha}{q^{4}(T-t)^{2}}+\frac{M}{(q-1)(T-t)}\right) \quad(t \in[0, T]) .
$$

In particular, from 4.19, $\rho_{0}$ is continuous and decreasing and satisfies 3.10). We can also check that

$$
\left|\frac{\left(\rho_{0}\right)^{2}}{\rho_{1}}\right| \leqslant C \quad((t \in[0, T]) .
$$

Proof. Applying Theorem 3.2 and Theorem 4.1, we deduce that for any $y_{0} \in \mathcal{H}^{-1}$ and $f \in L_{\rho_{1}}^{2}\left(0, T ; \mathcal{H}^{-3}\right)$, there exists $u \in L_{\rho_{0}}^{2}(0, T ; U)$ such that the solutions of

$$
\left\{\begin{array}{l}
y_{t}+y_{x x x x}+a y_{x x}=f, \quad x \in(0,1), \quad t \in(0, T) \\
y(t, 0)=0, \quad y(t, 1)=0, \quad t \in(0, T), \\
y_{x}(t, 0)=u(t), \quad y_{x}(t, 1)=0, \quad t \in(0, T), \\
y(0, x)=y_{0}(x), \quad x \in(0,1) .
\end{array}\right.
$$

satisfies

$$
\|y\|_{L_{\rho_{0}}^{2}\left(0, T ; \mathcal{H}^{1}\right)}^{2}+\left\|\frac{y}{\rho_{0}}\right\|_{C^{0}\left([0, T] ; \mathcal{H}^{-1}\right)}^{2}+\left\|y_{t}\right\|_{L_{\rho_{0}}^{2}\left(0, T ; \mathcal{H}^{-3}\right)}^{2}+\|u\|_{L_{\rho_{0}}^{2}(0, T)}^{2} \leqslant C\left(\left\|y_{0}\right\|_{\mathcal{H}^{-1}}^{2}+\|f\|_{L_{\rho_{1}}^{2}\left(0, T ; \mathcal{H}^{-3}\right)}^{2}\right) .
$$

Now, let us consider the map

$$
\mathcal{Z}: f \in L_{\rho_{1}}^{2}\left(0, T ; \mathcal{H}^{-3}\right) \mapsto-F(y) \in L_{\rho_{1}}^{2}\left(0, T ; \mathcal{H}^{-3}\right)
$$

where $(y, u)$ is the solution of 4.23 and where $F$ is defined by 4.8 . We show that $\mathcal{Z}$ is well-defined and that there exists $R$ such that

$$
\mathcal{Z}(B(0, R)) \subset B(0, R)
$$

where $B(0, R)$ is the closed ball of $L_{\rho_{1}}^{2}\left(0, T ; \mathcal{H}^{-3}\right)$ of radius $R>0$.

First using 4.8, we deduce that

$$
\|F(y)\|_{\mathcal{H}^{-2}} \leqslant C\|y\|_{\mathcal{H}^{1}}\|y\|_{\mathcal{H}^{-1}}
$$

Therefore, from 4.22 and 4.24

$$
\left.\|F(y)\|_{L_{\rho_{0}}^{2}\left(0, T ; \mathcal{H}^{-2}\right)} \leqslant C\left(\left\|y_{0}\right\|_{\mathcal{H}^{-1}}^{2}+\|f\|_{L_{\rho_{1}}^{2}(0, T ; \mathcal{H}}^{2-3}\right)\right) .
$$

Assume that

$$
R>\left\|y_{0}\right\|_{\mathcal{H}^{-1}}
$$

and take

$$
\|f\|_{L_{\rho_{1}}^{2}\left(0, T ; \mathcal{H}^{-3}\right)} \leqslant R
$$

Then,

$$
\|F(y)\|_{L_{\rho_{0}}^{2}\left(0, T ; \mathcal{H}^{-2}\right)} \leqslant 2 C R^{2} \leqslant R
$$

if we assume

$$
R=\frac{1}{4 C}
$$

We now show that $\mathcal{N}$ is a contraction on $B(0, R)$ : let us take $f^{(1)}, f^{(2)} \in B(0, R)$ and let us consider the corresponding solutions and controls $\left(y^{(1)}, u^{(1)}\right),\left(y^{(2)}, u^{(2)}\right)$. We write

$$
f:=f^{(1)}-f^{(2)}, \quad y:=y^{(1)}-y^{(2)}, \quad u:=u^{(1)}-u^{(2)} .
$$


We have

$$
\|y\|_{L_{\rho_{0}}^{2}\left(0, T ; \mathcal{H}^{1}\right)}^{2}+\left\|\frac{y}{\rho_{0}}\right\|_{C^{0}\left([0, T] ; \mathcal{H}^{-1}\right)}^{2}+\left\|y_{t}\right\|_{L_{\rho_{0}}^{2}\left(0, T ; \mathcal{H}^{-3}\right)}^{2}+\|u\|_{L_{\rho_{0}}^{2}(0, T)}^{2} \leqslant C\|f\|_{L_{\rho_{1}}^{2}\left(0, T ; \mathcal{H}^{-3}\right)}^{2} .
$$

From 4.8, we obtain

$$
\left\|F\left(y^{(1)}\right)-F\left(y^{(2)}\right)\right\|_{\mathcal{H}^{-2}} \leqslant C\left(\left\|y^{(1)}\right\|_{\mathcal{H}^{1}}+\left\|y^{(2)}\right\|_{\mathcal{H}^{1}}\right)\|y\|_{\mathcal{H}^{-1}}
$$

Combining 4.24 and 4.26 yields

$$
\left\|F\left(y^{(1)}\right)-F\left(y^{(2)}\right)\right\|_{L_{\rho_{0}}^{2}\left(0, T ; \mathcal{H}^{-2}\right)} \leqslant 2 C R\|f\|_{L_{\rho_{1}}^{2}\left(0, T ; \mathcal{H}^{-3}\right)} .
$$

and we deduce from 4.25 that $\mathcal{N}$ is a contraction.

\section{Controllability of the Kuramoto-Sivashinsky equation in 2D}

We show in this section the null-controllability of the Kuramoto-Sivashinsky equation in 2D (Theorem 1.4).

\subsection{The linear system}

We start by considering the null-controllability of a linear system associated with $(1.2)$ :

$$
\left\{\begin{array}{l}
y_{t}+\Delta^{4} y+a \Delta y=0, \quad x \in \Omega, t \in(0, T) \\
y(t)=0, \quad x \in \partial \Omega, \quad t \in(0, T) \\
\Delta y(t)=u 1_{\Gamma}, \quad x \in \partial \Omega, t \in(0, T), \\
y(0, x)=y_{0}(x), \quad x \in \Omega .
\end{array}\right.
$$

We define

$$
\begin{gathered}
\mathcal{D}\left(A_{0}\right)=\left\{y \in H^{4}(\Omega) ; y=\Delta y=0 \quad \text { on } \partial \Omega\right\}, \\
A_{0}: \mathcal{D}\left(A_{0}\right) \rightarrow L^{2}(\Omega), \quad y \mapsto \Delta^{4} y .
\end{gathered}
$$

The operator $A_{0}$ is self-adjoint, positive and with compact resolvent. We denote by $\lambda_{n}, n \in \mathbb{N}^{*}$, the eigenvalues of $A_{0}$. They are real positive and we denote by $\lambda_{1}$ the smallest one. Assume $a<\sqrt{\lambda_{1}}$. We also consider the operator

$$
\begin{gathered}
\mathcal{D}\left(A_{a}\right)=\mathcal{D}\left(A_{0}\right), \\
A_{a}: \mathcal{D}\left(A_{0}\right) \rightarrow L^{2}(\Omega), \quad y \mapsto \Delta^{4} y+a \Delta y .
\end{gathered}
$$

The operator $A_{a}$ is self-adjoint and $-A_{a}$ is the generator of a semi-group $\left(e^{-t A_{a}}\right)_{t \geqslant 0}$.

In what follows, we consider $\lambda_{\#} \in \rho\left(A_{a}\right)$ such that $A_{\#}:=\lambda_{\#}+A_{a}$ is positive and we set

$$
\mathcal{H}^{s}:=\mathcal{D}\left(A_{\#}^{s / 4}\right) \quad(s \geqslant 0), \quad \mathcal{H}^{-s}:=\mathcal{D}\left(A_{\#}^{s / 4}\right)^{\prime} \quad(s \geqslant 0),
$$

where $\mathcal{X}^{\prime}$ denotes the dual of $\mathcal{X}$ with respect to the space $L^{2}(\Omega)$.

We can consider $A_{a}$ as an operator

$$
A_{a}: \mathcal{H}^{s+4} \rightarrow \mathcal{H}^{s}, \quad(s \in \mathbb{R})
$$

In particular,

$$
A_{0}: \mathcal{H}^{s+4} \rightarrow \mathcal{H}^{s}, \quad(s \in \mathbb{R})
$$

is an isomorphism. 
In order to obtain a weak formulation for system (5.1), we multiply formally the first equation of (5.1) by $\varphi \in \mathcal{H}^{4}$. After some integrations by parts, we obtain

$$
\frac{d}{d t} \int_{\Omega} y \varphi d x+\int_{\Omega} y A_{a} \varphi d x=\int_{\Gamma} u \frac{\partial \varphi}{\partial \nu} d \gamma
$$

In order to write the corresponding control operator, we use a classical approach and define the operator $D_{0}: U=L^{2}(\Gamma) \rightarrow \mathcal{H}^{2}$ as follows: $D_{0} u=y$ is the solution of

$$
\left\{\begin{array}{l}
\Delta^{4} y=0 \quad \text { in } \Omega \\
y=0 \text { on } \partial \Omega \\
\Delta y=u 1_{\Gamma} \quad \text { on } \partial \Omega
\end{array}\right.
$$

More precisely, $D_{0} u$ is the unique element of $\mathcal{H}^{2}$ such that

$$
\left\langle D_{0} u, f\right\rangle_{\mathcal{H}^{2}, \mathcal{H}^{-2}}=\int_{\Gamma} u \frac{\partial}{\partial \nu}\left(A_{0}^{-1} f\right) d \gamma .
$$

Since $A_{0}^{-1}\left(\mathcal{H}^{-2}\right)=\mathcal{H}^{2}, D_{0} u$ is well-defined.

Then, we define the operator $B_{0}$ by

$$
B_{0}:=-A_{0} D_{0}: U \rightarrow \mathcal{H}^{-2}
$$

One can check that for all $\varphi \in \mathcal{H}^{2}$ and $u \in U$,

$$
\left\langle B_{0} u, \varphi\right\rangle_{\mathcal{H}^{-2}, \mathcal{H}^{2}}=\int_{\Gamma} u \frac{\partial \varphi}{\partial \nu} d \gamma
$$

As a consequence, (5.1) writes as

$$
\dot{y}=-A_{0} y-a A_{0}^{1 / 2} y+B_{0} u, \quad y(0)=y_{0} .
$$

Considering $A_{\#}$ as an operator from $\mathcal{H}^{4}$ onto $\mathcal{H}$, we deduce that

$$
B_{0}: U \rightarrow D\left(A_{\#}^{1 / 2}\right)^{*}
$$

and from (5.11), its adjoint satisfies

$$
C_{0}:=B_{0}^{*}: \mathcal{H}^{2} \rightarrow U, \varphi \mapsto \frac{\partial \varphi}{\partial \nu \mid \Gamma}
$$

We are now in position to prove the null-controllability of (5.1)

Theorem 5.1. Assume $a<\sqrt{\lambda_{1}}$ and assume conditions 1) or 2) of Theorem 1.4. For $y_{0} \in \mathcal{H}$, there exists $u \in$ $L^{2}(0, T ; U)$ such that the solution of $(5.1)$ satisfies $y(T)=0$. Moreover, there exists an operator $L_{T} \in \mathcal{L}(\mathcal{H}, U)$ such that $u$ can be given by $u=L_{T}\left(y_{0}\right)$. We have

$$
\left\|L_{T}\right\|_{\mathcal{L}\left(\mathcal{H}^{-1}, \mathbb{R}\right)} \leqslant K(T)
$$

with

$$
K(T):=C e^{M / T}
$$

for some positive constants $C, M$. 
Proof. In order to obtain the controllability of (5.12), we use two results on the plate equations obtained by 13 . and [20] (see also [17] and Remark 7.5.6 in [21]): assume one of the conditions of Theorem 1.4 then the system

$$
\left\{\begin{array}{l}
\ddot{w}=-A_{0} w \\
y=C_{0} \dot{w}
\end{array}\right.
$$

is exactly observable in $\mathcal{H}^{3} \times \mathcal{H}^{1}$.

Then using Theorem 3.3 , we deduce that

$$
\left\{\begin{array}{l}
\ddot{w}=-A_{0} w+a A_{0}^{1 / 2} w \\
y=C_{0} \dot{w} .
\end{array}\right.
$$

is exactly observable in $\mathcal{H}^{3} \times \mathcal{H}^{1}$ for $a<\sqrt{\lambda_{1}}$.

Moreover, one has (see, for instance [21, Proposition 3.6.9]) that the eigenvalues $\left(\lambda_{n}(a)\right)_{n \in \mathbb{N}^{*}}$ of $A_{a}$ satisfies

$$
c_{1} n^{2} \leqslant \lambda_{n}(a) \leqslant c_{2} n^{2} \quad\left(n \geqslant n_{0}\right) .
$$

for some positive constants $c_{1}$ and $c_{2}$ and some $n_{0} \geqslant 1$. We can apply Theorem 3.1

$$
\left\{\begin{array}{l}
\dot{z}=-A_{0} z+a A_{0}^{1 / 2} z \\
y=C_{0} \dot{z} .
\end{array}\right.
$$

is final state state observable in $\mathcal{H}^{1}$ for any time $T>0$ with a cost $K$ satisfying (3.5). This means that

$$
\left\|e^{-A_{a} T} \varphi_{0}\right\|_{\mathcal{H}^{1}} \leqslant K(T)\left(\int_{0}^{T}\left\|B_{0}^{*} e^{-A_{a} t} \varphi_{0}\right\|_{U}^{2} d t\right)^{1 / 2} \quad\left(\varphi_{0} \in \mathcal{H}^{2}\right)
$$

and there exist $C$ and $M$ such that

$$
K(T):=C e^{M / T}
$$

This implies in particular

$$
\left\|e^{-A_{a} T} \varphi_{0}\right\|_{\mathcal{H}} \leqslant K(T)\left(\int_{0}^{T}\left\|B_{0}^{*} e^{-A_{a} t} \varphi_{0}\right\|_{U}^{2} d t\right)^{1 / 2} \quad\left(\varphi_{0} \in \mathcal{H}^{2}\right) .
$$

From standard duality arguments, we deduce that 5.1 is null-controllable for any time $T>0$ in $\mathcal{H}$ : for any $y_{0} \in \mathcal{H}$, there exists $u \in L^{2}(0, T ; U)$ with

$$
\|u\|_{L^{2}(0, T ; U)} \leqslant K(T)\left\|y_{0}\right\|_{\mathcal{H}}
$$

such that $y(T)=0$.

\subsection{Proof of Theorem 1.4}

We are now in position to prove Theorem 1.4. The proof is similar to the proof of Theorem 1.1 we take $q, \alpha$, $\rho_{1}$ and $\rho_{0}$ as in 4.19, 4.20, 4.21). In particular, 4.22) holds.

Proof. Applying Theorem 3.2 and Theorem 5.1. we deduce that for any $y_{0} \in \mathcal{H}$ and $f \in L_{\rho_{1}}^{2}\left(0, T ; \mathcal{H}^{-2}\right)$, there exists $u \in L_{\rho_{0}}^{2}(0, T ; U)$ such that the solutions of

$$
\left\{\begin{array}{l}
y_{t}+\Delta^{4} y+a \Delta y=f, \quad x \in \Omega, t \in(0, T) \\
y(t)=0, \quad x \in \partial \Omega, t \in(0, T) \\
\Delta y(t)=u 1_{\Gamma}, \quad x \in \partial \Omega, t \in(0, T) \\
y(0, x)=y_{0}(x), \quad x \in \Omega
\end{array}\right.
$$


satisfies

$$
\|y\|_{L_{\rho_{0}}^{2}\left(0, T ; \mathcal{H}^{2}\right)}^{2}+\left\|\frac{y}{\rho_{0}}\right\|_{C^{0}([0, T] ; \mathcal{H})}^{2}+\left\|y_{t}\right\|_{L_{\rho_{0}}^{2}\left(0, T ; \mathcal{H}^{-2}\right)}^{2}+\|u\|_{L_{\rho_{0}}^{2}(0, T ; U)}^{2} \leqslant C\left(\left\|y_{0}\right\|_{\mathcal{H}}^{2}+\|f\|_{L_{\rho_{1}}^{2}\left(0, T ; \mathcal{H}^{-2}\right)}^{2}\right) .
$$

Now, let us consider the map

$$
\mathcal{Z}: f \in L_{\rho_{1}}^{2}\left(0, T ; \mathcal{H}^{-2}\right) \mapsto-F(y) \in L_{\rho_{1}}^{2}\left(0, T ; \mathcal{H}^{-2}\right)
$$

where $(y, u)$ is the solution of $(5.19)-5.20)$ and where $F$ is defined by:

$$
F(y)=-\frac{1}{2}|\nabla y|^{2}
$$

We show that $\mathcal{Z}$ is well-defined and that there exists $R$ such that

$$
\mathcal{Z}(B(0, R)) \subset B(0, R)
$$

where $B(0, R)$ is the closed ball of $L_{\rho_{1}}^{2}\left(0, T ; \mathcal{H}^{-2}\right)$ of radius $R>0$.

First using the Sobolev embedding $\mathcal{H}^{2} \subset L^{\infty}(\Omega)$ and an interpolation argument, we deduce that

$$
\|F(y)\|_{\mathcal{H}^{-2}} \leqslant C\|y\|_{\mathcal{H}^{1}}^{2} \leqslant C\|y\|_{\mathcal{H}^{2}}\|y\|_{\mathcal{H}} .
$$

Therefore, from 4.22 and 5.20

$$
\|F(y)\|_{L_{\rho_{1}}^{2}\left(0, T ; \mathcal{H}^{-2}\right)} \leqslant C\left(\left\|y_{0}\right\|_{\mathcal{H}}^{2}+\|f\|_{L_{\rho_{1}}^{2}\left(0, T ; \mathcal{H}^{-2}\right)}^{2}\right) .
$$

Using this estimate, we can apply the Banach fixed point theorem as in the proof of Theorem 1.1 .

\section{References}

[1] Eduardo Cerpa. Exact controllability of a nonlinear Korteweg-de Vries equation on a critical spatial domain. SIAM J. Control Optim., 46(3):877-899 (electronic), 2007.

[2] Eduardo Cerpa. Null controllability and stabilization of the linear Kuramoto-Sivashinsky equation. Commun. Pure Appl. Anal., 9(1):91-102, 2010.

[3] Eduardo Cerpa and Emmanuelle Crépeau. Boundary controllability for the nonlinear Korteweg-de Vries equation on any critical domain. Ann. Inst. H. Poincaré Anal. Non Linéaire, 26(2):457-475, 2009.

[4] Eduardo Cerpa and Alberto Mercado. Local exact controllability to the trajectories of the 1-D KuramotoSivashinsky equation. J. Differential Equations, 250(4):2024-2044, 2011.

[5] Panagiotis D. Christofides and Antonios Armaou. Global stabilization of the Kuramoto-Sivashinsky equation via distributed output feedback control. Systems Control Lett., 39(4):283-294, 2000.

[6] Nicolae Cîndea and Marius Tucsnak. Local exact controllability for Berger plate equation. Math. Control Signals Systems, 21(2):93-110, 2009.

[7] Jean-Michel Coron and Qi Lü. Fredholm transform and local rapid stabilization for a Kuramoto-Sivashinsky equation. J. Differential Equations, 259(8):3683-3729, 2015.

[8] Szymon Dolecki and David L. Russell. A general theory of observation and control. SIAM J. Control Optimization, 15(2):185-220, 1977.

[9] C. Foias, B. Nicolaenko, G. R. Sell, and R. Temam. Inertial manifolds for the Kuramoto-Sivashinsky equation and an estimate of their lowest dimension. J. Math. Pures Appl. (9), 67(3):197-226, 1988. 
[10] Changbing $\mathrm{Hu}$ and Roger Temam. Robust control of the Kuramoto-Sivashinsky equation. Dyn. Contin. Discrete Impuls. Syst. Ser. B Appl. Algorithms, 8(3):315-338, 2001.

[11] M. S. Jolly, R. Rosa, and R. Temam. Evaluating the dimension of an inertial manifold for the KuramotoSivashinsky equation. Adv. Differential Equations, 5(1-3):31-66, 2000.

[12] Yoshiki Kuramoto. Diffusion-induced chaos in reaction systems. Progress of Theoretical Physics Supplement, 64:346-367, 1978.

[13] G. Lebeau. Contrôle de l'équation de Schrödinger. J. Math. Pures Appl. (9), 71(3):267-291, 1992.

[14] Yuning Liu, Takéo Takahashi, and Tucsnak Marius. Single input controllability of a simplified fluidstructure interaction model. ESAIM Control Optim. Calc. Var., 2013.

[15] B. Nicolaenko, B. Scheurer, and R. Temam. Some global dynamical properties of the Kuramoto-Sivashinsky equations: nonlinear stability and attractors. Phys. D, 16(2):155-183, 1985.

[16] B. Nicolaenko, B. Scheurer, and R. Temam. Some global dynamical properties of a class of pattern formation equations. Comm. Partial Differential Equations, 14(2):245-297, 1989.

[17] K. Ramdani, T. Takahashi, G. Tenenbaum, and M. Tucsnak. A spectral approach for the exact observability of infinite-dimensional systems with skew-adjoint generator. J. Funct. Anal., 226(1):193-229, 2005.

[18] David L. Russell. A unified boundary controllability theory for hyperbolic and parabolic partial differential equations. Studies in Appl. Math., 52:189-211, 1973.

[19] GI Sivashinsky. Nonlinear analysis of hydrodynamic instability in laminar flamesi. derivation of basic equations. Acta astronautica, 4(11):1177-1206, 1977.

[20] G. Tenenbaum and M. Tucsnak. Fast and strongly localized observation for the Schrödinger equation. Trans. Amer. Math. Soc., 361(2):951-977, 2009.

[21] Marius Tucsnak and George Weiss. Observation and control for operator semigroups. Birkhäuser Advanced Texts: Basler Lehrbücher. [Birkhäuser Advanced Texts: Basel Textbooks]. Birkhäuser Verlag, Basel, 2009. 